\title{
Diagnosis of Primary Cardiac T-cell Lymphoma: Feasibility and Safety of Endomyocardial Biopsy Guided by Pre-acquired Cardiovascular Magnetic Resonance
}

\author{
Andrea Avella ${ }^{1}$, Carla Giordano ${ }^{2}$, Vitaliano Buffa ${ }^{3}$, Piergiuseppe De Girolamo ${ }^{1}$, Massimo Uguccioni ${ }^{1}$ \\ ${ }^{1}$ Cardiology Division and Cardiac Arrhythmia Unit, St. Camillo-Forlanini Hospital, Rome, Italy \\ ${ }^{2}$ Department of Radiology, Oncology and Pathology, Sapienza University, Rome, Italy \\ ${ }^{3}$ Cardiovascular Imaging Unit, St. Camillo-Forlanini Hospital, Rome, Italy
}

Received: $27 / 02 / 2021$

Accepted: 02/03/2021

Published: $25 / 03 / 2021$ How to cite this article: Avella A, Giordano C, Buffa V, De Girolamo P, Uguccioni M. Diagnosis of primary cardiac T-cell lymphoma: feasibility and safety of
endomyocardial biopsy guided by pre-acquired cardiovascular magnetic resonance. EJCRIM 2021;8: doi:10.12890/2021_002427.

Conflicts of Interests: The Authors declare that there are no competing interests.

This article is licensed under a Commons Attribution Non-Commercial 4.0 License

\section{ABSTRACT}

Background: Multimodality imaging of a cardiac mass lesion may raise suspicion of a primitive cardiac lymphoma (PCL). However, a definitive diagnosis requires histopathological confirmation.

Methods: This report describes the methodology we used to perform biopsy sampling of a cardiac mass lesion affecting a 45-year-old man. In order to increase endomyocardial biopsy diagnostic accuracy, we used pre-acquired cardiac magnetic resonance (CMR) images to guide the bioptome on a cardiac site overtly infiltrated by the suspected tumour. The right ventricular outflow tract was identified as the target site for biopsy sampling. To reduce the risk of the procedure, the biopsy was performed at a safe distance from the tip of a diagnostic quadripolar catheter positioned at the level of the pulmonary valve, previously identified by pacing manoeuvres. The reported approach demonstrated safety and diagnostic accuracy, allowing the identification of an extremely rare PCL subtype of T-cell origin.

Conclusion: Biopsy sampling of a suspected tumour may be safely and accurately performed using pre-acquired CMR images to guide the bioptome on the target site.

\section{LEARNING POINTS}

- Multimodal imaging techniques capable of tissue characterization may raise suspicion of a primary cardiac lymphoma (PCL).

- However, the final diagnosis of PCL can be confirmed only by histological examination of a tissue sample.

- Biopsy sampling of the mass lesion may be accurately guided by previously acquired cardiac magnetic resonance images.

\section{KEYWORDS}

Cardiac tumour, cardiac magnetic resonance, right-sided catheterization, endomyocardial biopsy

\section{INTRODUCTION}

Endomyocardial biopsy (EMB) is a valuable tool for studying intracardiac masses suspicious for tumours. Nonetheless, the diagnostic accuracy of the standard biopsy procedure, with fluoroscopic guidance, may be significantly limited by sampling errors. Transoesophageal and intracardiac echocardiography have been successfully used to guide the biopsy procedure in patients with suspected cardiac tumours. Nevertheless, there has been no consensus on a standardized approach. 
In order to increase the precision of biopsy sampling, while reducing the risk of complications, we used pre-acquired cardiac magnetic resonance (CMR) images to guide the bioptome on a cardiac target site overtly infiltrated by a suspected tumour. The reported approach demonstrated safety and diagnostic accuracy, allowing the identification of an extremely rare primary cardiac malignancy.

\section{CASE DESCRIPTION}

A 45-year-old man was referred to our hospital for diagnosis confirmation of a suspected cardiac tumour detected incidentally. The patient had remained in a stable clinical condition until 5 months before admission, when a routine cardiological assessment for recreational sports activity had revealed on ECG de novo significant T wave alterations (Fig. 1), not associated with remarkable findings on physical examination. The patient's medical and family histories were negative for cardiovascular disease and the patient was not complaining of cardiological or constitutional symptoms. Afterwards, an outpatient non-invasive evaluation was initiated.

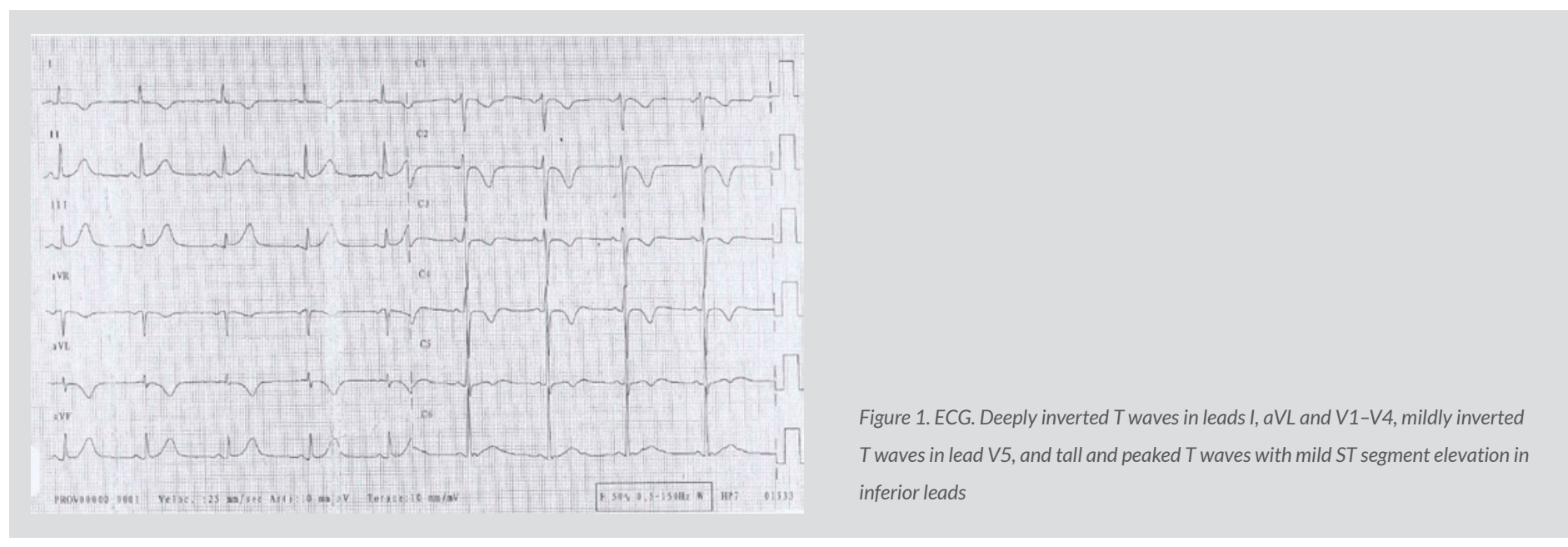

Laboratory work-up, including an immunological profile, and chest radiograph did not reveal any abnormal findings. Transthoracic echocardiography showed increased thickness and echogenicity of right ventricular outflow tract (RVOT) and basal free wall. A small circumferential pericardial effusion, with no signs of haemodynamic compromise, was also reported. CMR revealed an epicardial thickened mass lesion adherent to the anterior wall of both ventricles and invading the interventricular sulcus (Fig. 2). The anterior left ventricular wall and anterior interventricular septum were both infiltrated by the abnormal tissue. Apparent full-thickness wall replacement of the RVOT was also observed. Relative to myocardium, the mass lesion appeared markedly hyperintense on T2-weighted TIRM images and mildly hyperintense on True FISP cine images, also showing a large interventricular focus of late gadolinium enhancement. Full body computed tomography (CT) was unremarkable, excluding both metastatic disease and direct cardiac infiltration from adjacent malignancy.

Multimodality imaging results were consistent with a primary cardiac tumour with overt biventricular involvement. Hence, the patient, still asymptomatic, was admitted to our institution for diagnosis confirmation. At presentation, he was haemodynamically stable and afebrile; vital signs were normal, cardiopulmonary examination was unremarkable, and there was no lymphadenopathy. The remainder of his physical examination also showed no abnormalities. The results of a complete blood count, coagulation profile and comprehensive metabolic panel were within normal limits.

We planned an EMB guided by CMR images. Transmural RVOT infiltration was identified as a suitable target site for biopsy sampling. After obtaining informed written consent from the patient, a $6 \mathrm{~F}$ diagnostic quadripolar catheter was inserted via the inferior vena cava approach. Then, under fluoroscopic guidance, the catheter was positioned in the RV infundibulum with its distal portion at the level of the pulmonary valve, identified by pacing manoeuvres. Subsequently, by utilizing an $8.5 \mathrm{~F}$ steerable long introducer, a disposable bioptome was also advanced in the RVOT and oriented towards the target region, previously identified on CMR images. Four specimens were obtained from the RVOT anterior and septal walls (Fig. 3), while keeping the bioptome at a safe distance from the proximal bipole of the quadripolar catheter positioned at the level of the pulmonary valve.

The biopsy procedure was successfully performed with no complications. All specimens were fixed in $10 \%$ buffered formalin, processed and stained with haematoxylin and eosin. Serial sections were subjected to immunohistochemical staining with antibodies against CD3, CD20, CD10, Bcl6, Bcl2, Pax 5, CD5, cyclin D1, CD4, CD8, CD30 and Ki67 (Dako, Glostrup, Denmark). 

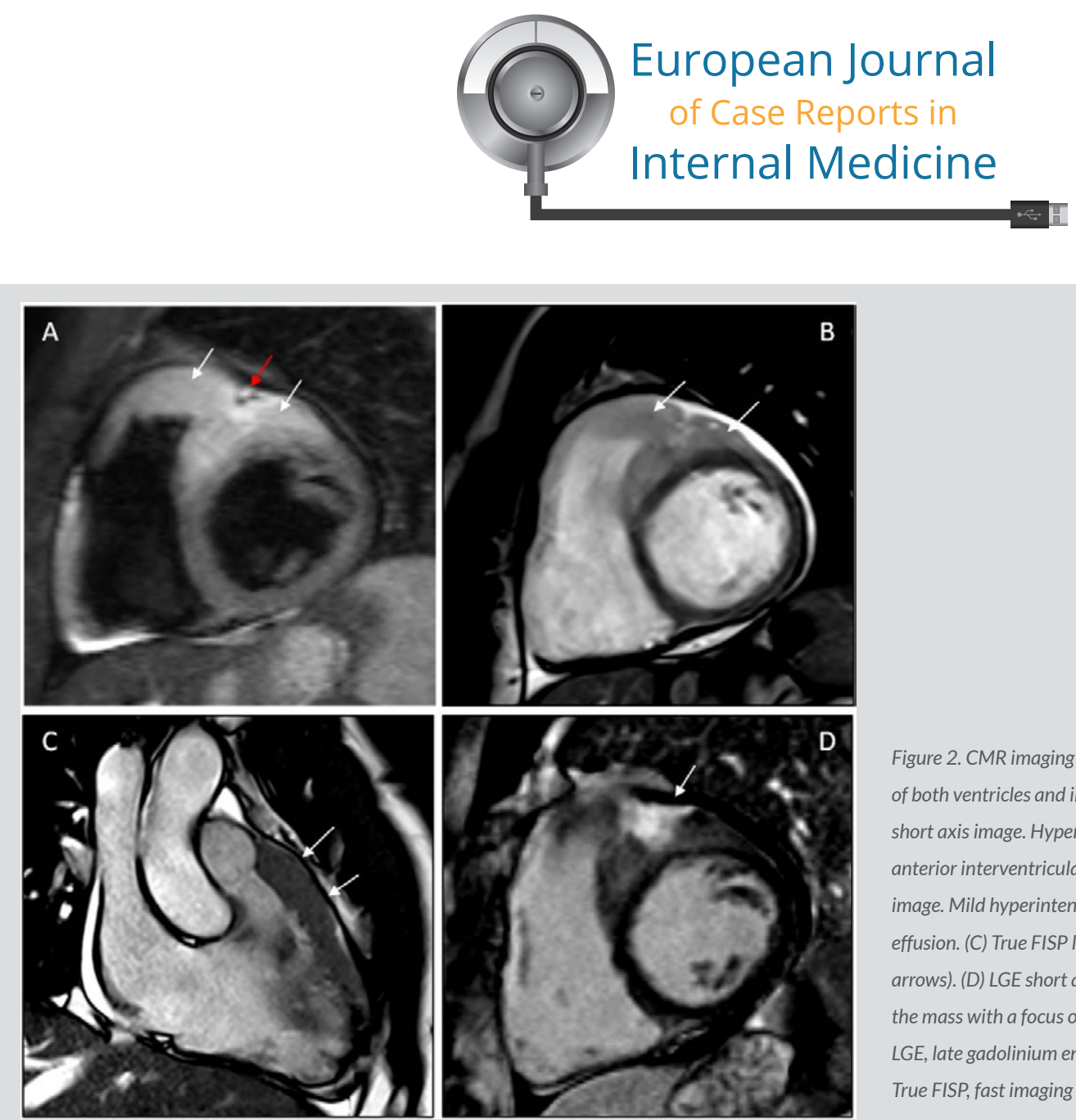

Figure 2. CMR imaging showing a mass lesion adherent to the anterior wall of both ventricles and invading the interventricular sulcus. (A) T2W TIRM short axis image. Hyperintensity of the mass (white arrows), encasing the anterior interventricular coronary artery (red arrow). (B) True FISP short axis image. Mild hyperintensity of the mass (white arrows), with a small pericardial effusion. (C) True FISP long axis image. Anterior extension of the mass (white arrows). (D) LGE short axis image. Mild and inhomogeneous hyperintensity of the mass with a focus of LGE (white arrow). CMR, cardiac magnetic resonance; LGE, late gadolinium enhancement; TIRM, turbo inversion recovery magnitude; True FISP, fast imaging with steady-state free precession technique
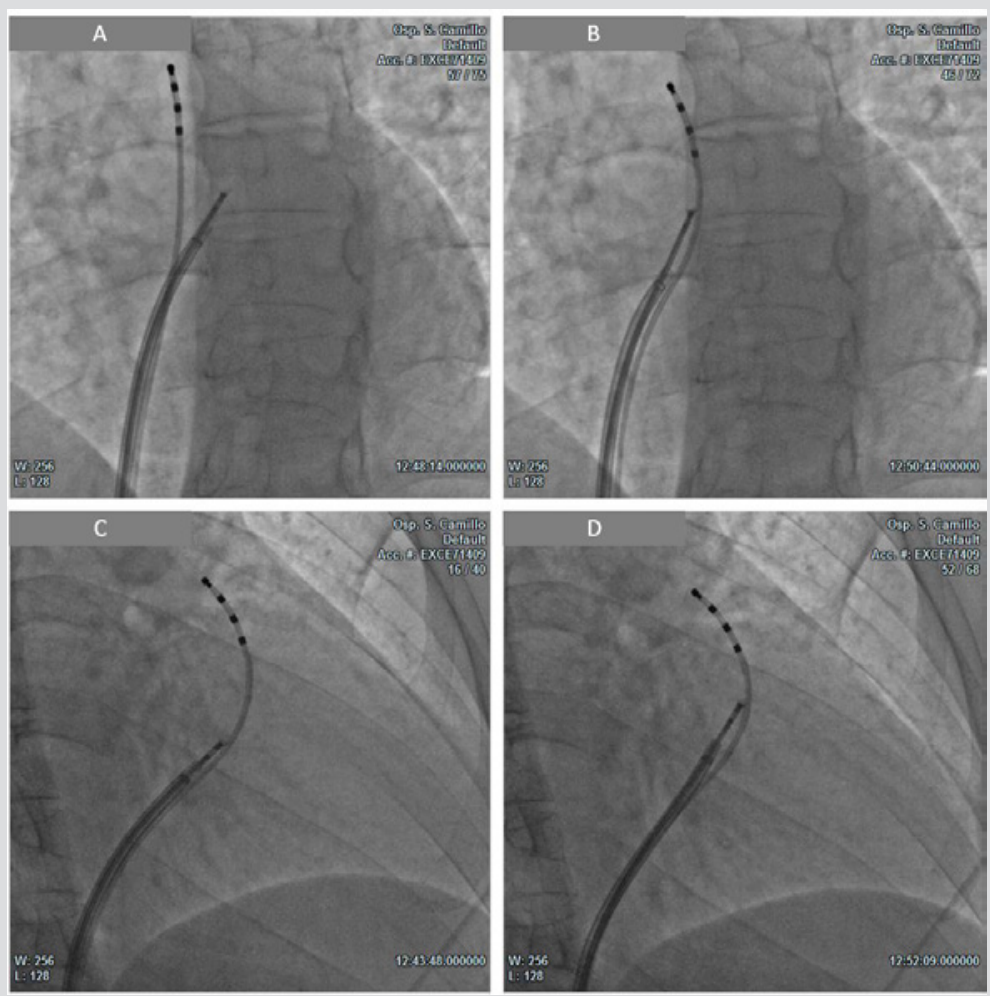

Figure 3. Biopsy procedure. $L A O(A, B)$ and $R A O(C, D)$ fluoroscopic projections: bioptome collecting specimens from the right ventricular outflow tract at $a$ safe distance from the quadripolar catheter tip positioned at the level of the pulmonary valve. $L A O$, left anterior oblique; $R A O$, right anterior oblique

Two of the four samples obtained from the RVOT showed diffuse proliferation of small-medium sized lymphocytes almost completely replacing the myocardium (Fig. 4A,B). Lymphocytes strongly expressed the T-cell markers CD3 and CD5 (Fig. 4C), with a CD4:CD8 ratio of 1:2. Focal aberrant expression of CD20 was observed. Staining with the other antibodies tested, including the B-cell marker Pax5, was negative. The proliferation index evaluated by Ki67 was about 30\%. The two remaining samples showed normal myocardium (Fig. 4D). 
According to the clinical, morphological and immunohistochemical features, a diagnosis of primitive cardiac T-cell lymphoma was established. Subsequently, the patient was transferred to the haematology division at another institution where a multichemotherapy regimen was started. After six cycles of treatment with CHOP (cyclophosphamide, doxorubicin, vincristine and prednisone), repetition of CMR documented a significant regression of the mass lesion (Fig. 5). Currently, 12 months after the initial clinical assessment, the patient has preserved biventricular function and a normal functional capacity.

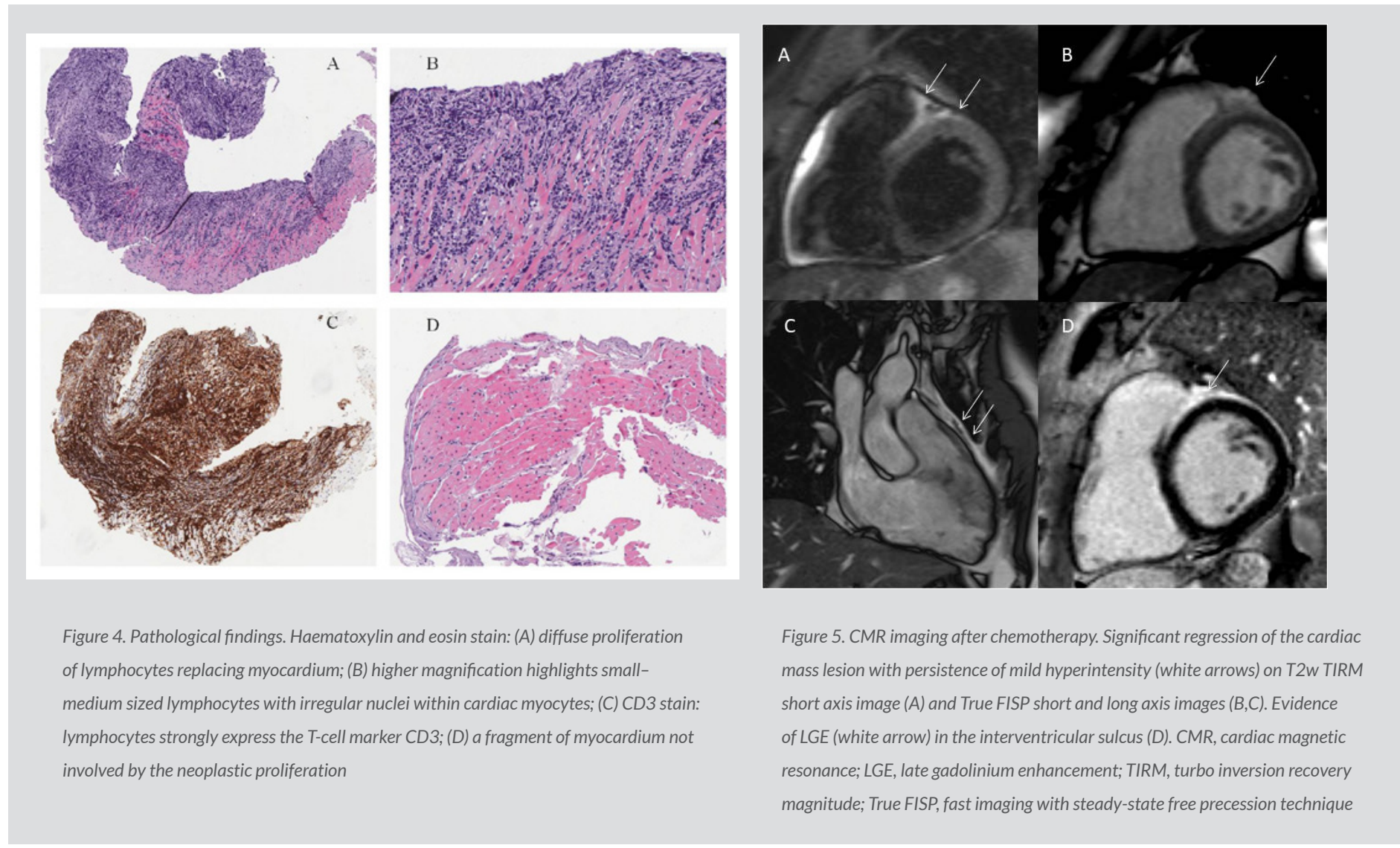

\section{DISCUSSION}

Primary cardiac lymphoma (PCL) is a very rare malignancy, typically of a non-Hodgkin's type, accounting for 1-2\% of primary cardiac tumours and $0.5 \%$ of extra-nodal lymphomas ${ }^{[1]}$. The right atrium is the most commonly affected heart chamber, followed by the right ventricle, left atrium and left ventricle. PCL is often multifocal, involving more than one heart chamber in $>75 \%$ of cases ${ }^{[1]}$.

Presenting signs and symptoms of PCL are non-specific and may vary according to the heart site involved. The most common clinical manifestations include dyspnoea, constitutional symptoms, chest pain, congestive heart failure, pericardial effusion and arrhythmias ${ }^{[1,2]}$.

Histologically, nearly all cases of PCL are of B-cell origin, with diffuse large B-cell lymphoma being the most common subtype ${ }^{[1,2]}$. Conversely, we report an extremely rare PCL subtype of T-cell origin, described in only a handful of case reports over the last two decades ${ }^{[3-6]}$. In the largest literature review concerning PCL, only 5 of 197 cases were of T-cell origin ${ }^{[1]}$.

PCL can be rapidly fatal if left untreated, hence timely diagnosis is critical to improve outcome. Chemotherapy, alone or in combination with radiotherapy, seems to provide the best results ${ }^{[1,2]}$. However, palliative surgery may be required when haemodynamics are compromised by the tumour ${ }^{[7]}$.

The diagnosis of PCL is often challenging; hence, when the initial diagnostic work-up raises suspicion of a cardiac malignancy, a thorough evaluation with multimodal imaging techniques, capable of tissue characterization, is mandatory. Contrast CT, CMR as well as PET/CT may all be used for initial diagnosis and staging ${ }^{[8]}$. Nevertheless, PCL diagnosis confirmation requires histopathological analysis of specimens obtained through pericardiocentesis, endomyocardial or surgical biopsy.

Fluoroscopy-guided EMB may be useful for definitive characterization of cardiac tumours. Nevertheless, its diagnostic accuracy may be significantly limited by sampling errors. Transoesophageal and intracardiac echocardiography have been successfully used to improve 
the diagnostic accuracy and safety of EMB ${ }^{[9,10]}$. However, these ultrasound-based techniques usually require deep sedation or general anaesthesia and imply additional technical skills and costs.

In the reported case, targeted biopsy sampling of the mass lesion was obtained by combining real-time fluoroscopic guidance with previously acquired CMR images. The bioptome was guided towards the region of interest (the RVOT) by comparing the actual fluoroscopic image on the screen with the stored CMR images. The final position of the bioptome was adjusted step-by-step on the target site while keeping the tip of the device at a safe distance from the quadripolar catheter tip crossing the pulmonary valve.

To the best of our knowledge, this is the first report concerning CMR-guided EMB of a PCL. Our approach demonstrated safety and diagnostic accuracy, allowing the identification of an extremely rare PCL subtype of T-cell origin.
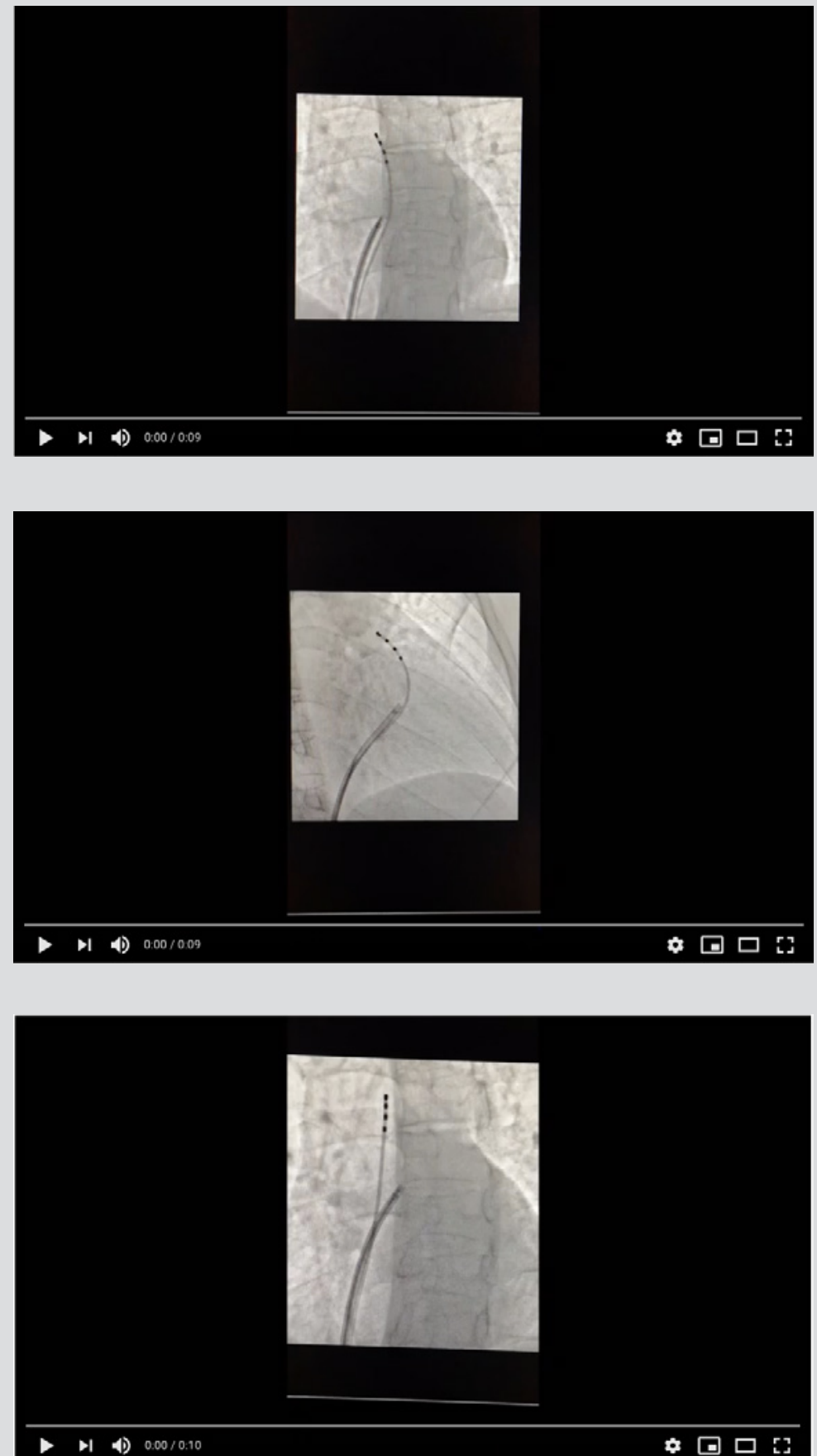

Video 1. Right ventricle outflow tract (RVOT) biopsy sampling watch the video: $h$ ttps://youtu.be/fMC4a5wAr4M

Video 2. Right ventricle outflow tract (RVOT) biopsy sampling watch the video: https://youtu.be/rmo_zv4H2pO

Video 3. Right ventricle outflow tract (RVOT) biopsy sampling watch the video: $h$ ttps://youtu.be/tPEbGXVMURc 


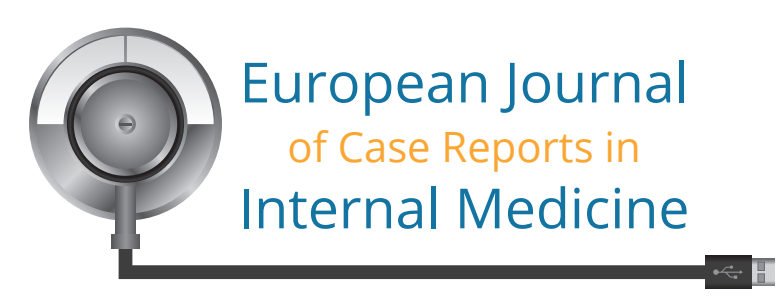

\section{CONCLUSION}

Multimodality imaging of a cardiac mass lesion may raise suspicion of a PCL. However, a definitive diagnosis requires histopathological confirmation. Biopsy sampling of the suspected tumour may be safely and accurately performed using pre-acquired CRM images to guide the bioptome on the target site.

\section{REFERENCES}

1. Petrich A, Cho SI, Billett H. Primary cardiac lymphoma: an analysis of presentation, treatment and outcome patterns. Cancer 2011;117:581-589.

2. Carras S, Berger F, Chalabreysse L, Callet-Bauchut E, Cordier J-F, Salles G, et al. Primary cardiac lymphoma: diagnosis, treatment and outcome in a modern series. Hematol Oncol 2017;35(4):510-519.

3. Werner D, Schmeisser A, Daniel WG. Images in cardiology: primary cardiac lymphoblastic T cell lymphoma. Heart 2001;86(6):618.

4. Patel J, Melly L, Sheppard MN. Primary cardiac lymphoma: B- and T-cell cases at a specialist UK centre. Ann Oncol 2010;21(5):1041-1045

Li B, Li R, Wu B, Chen X, Ni Y, Li W. Primary cardiac T cell lymphoma. J Card Surg 2012;27:457-460.

Pirzada A, Connors S, Harris S, Adams C. Primary cardiac T cell lymphoma mimicking ST-elevation myocardial infarction. Cardiology 2017;138:259-263.

Jovanicius K, Salcius K, Meskauskas R, Valeviciene N, Tarutis V, Sirvydis V. Primary cardiac lymphoma: two cases and a review of literature. J Cardiothorac Surg 2015;10:138.

Bhattacharyya S, Khattar RS, Gujral DM, Senior R. Cardiac tumors: the role of cardiovascular imaging. Expert Rev Cardiovasc Ther 2014;12(1):37-43.

9. Burling F, Devlin G, Heald S. Primary cardiac lymphoma diagnosed with transesophageal echocardiography-guided endomyocardial biopsy. Circulation 2000;101(17):E179E181.

10. Zanobini M, Dello Russo A, Saccocci M, Conti S, De Camilli E, Vettor G, et al. Endomyocardial biopsy guided by intracardiac echocardiography as a key step in intracardiac mass diagnosis. BMC Cardiovasc Disord 2018;18(1):15 